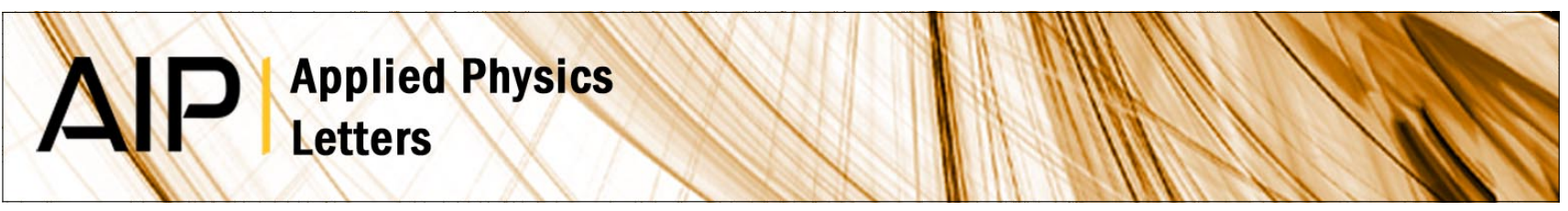

\title{
Current annealing and electrical breakdown of epitaxial graphene
}

S. Hertel, F. Kisslinger, J. Jobst, D. Waldmann, M. Krieger et al.

Citation: Appl. Phys. Lett. 98, 212109 (2011); doi: 10.1063/1.3592841

View online: http://dx.doi.org/10.1063/1.3592841

View Table of Contents: http://apl.aip.org/resource/1/APPLAB/v98/i21

Published by the American Institute of Physics.

\section{Related Articles}

Charging of nanostructured and partially reduced graphene oxide sheets

Appl. Phys. Lett. 101, 183109 (2012)

Temperature dependence of reversible switch-memory in electron field emission from ultrananocrystalline diamond

Appl. Phys. Lett. 101, 173116 (2012)

Controlling the current flux in magnetic-barrier induced graphene waveguide

Appl. Phys. Lett. 101, 163104 (2012)

Resonant tunneling through double barrier graphene systems: A comparative study of Klein and non-Klein tunneling structures

J. Appl. Phys. 112, 073711 (2012)

Electronic scattering of pseudo-magnetic field induced by local bump in graphene

J. Appl. Phys. 112, 073710 (2012)

\section{Additional information on Appl. Phys. Lett.}

Journal Homepage: http://apl.aip.org/

Journal Information: http://apl.aip.org/about/about_the_journal

Top downloads: http://apl.aip.org/features/most_downloaded

Information for Authors: http://apl.aip.org/authors

\section{ADVERTISEMENT}
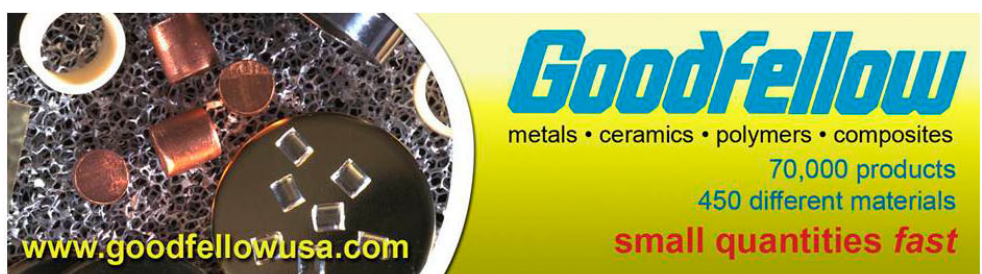


\title{
Current annealing and electrical breakdown of epitaxial graphene
}

\author{
S. Hertel, F. Kisslinger, J. Jobst, D. Waldmann, M. Krieger, and H. B. Weber ${ }^{\text {a) }}$ \\ Lehrstuhl für Angewandte Physik, Universität Erlangen-Nürnberg, Staudtstraße 7, 91058 Erlangen, Germany
}

(Received 22 February 2011; accepted 15 April 2011; published online 26 May 2011)

\begin{abstract}
We report on epitaxial graphene on silicon carbide at high current densities. We observe two distinguished regimes, and a final breakdown. First for low current densities the conductance is enhanced due to desorption of adsorbates. Second with increasing bias the sample locally starts to glow and is strongly heated. The silicon carbide material decomposes, graphitic material is formed and thus additional current paths are created. The graphene layer breaks down, which is, however, not visible in high bias data. The final breakdown is a self-amplifying process resulting in a locally destroyed sample but surprisingly with better conductance than the original sample. (c) 2011 American Institute of Physics. [doi:10.1063/1.3592841]
\end{abstract}

Graphene has rich perspectives for electronic applications. Due to the high charge carrier mobility graphene may play a role for future electronics. Sensor concepts are envisaged which benefit from the ultimate surface-to-volume ratio. The high optical transmission and low resistivity of graphene suggest an application as transparent electrodes. A large applied research effort is dedicated to these properties. We investigate epitaxial graphene, grown on the silicon face of semi-insulating silicon carbide $(\mathrm{SiC})$ by thermal decomposition at $T=1700{ }^{\circ} \mathrm{C}$ under argon atmosphere. ${ }^{1}$ The graphene layer is charged with an electron density of $n \approx 10^{13} \mathrm{~cm}^{-2}$, has high structural quality and high charge carrier mobility. The material is predominantly singlelayered, and shows graphene's specific quantum Hall effect. ${ }^{2}$ The substrate $\mathrm{SiC}$ is also an exceptional material providing chemical inertness and high mechanical hardness. In particular, it withstands high electric fields even at high temperatures. ${ }^{3}$ If appropriately doped, the material can also have high current (HC)-carrying capabilities. In this letter, we report on the $\mathrm{HC}$ behavior of epitaxial graphene. It is well known that at $\mathrm{HC}$ densities most metals experience an electromigration breakdown. Also for exfoliated graphene, an electromigration breakdown was reported ${ }^{4}$ at sheet current densities of $\approx 1.6 \mathrm{~mA} / \mu \mathrm{m}$. Assuming the van der Waals thickness of $0.335 \mathrm{~nm}$ as sheet thickness this corresponds to $\approx 5 \times 10^{8} \mathrm{~A} / \mathrm{cm}^{2}$. Similarly, experiments on carbon nanotubes ${ }^{5,6}$ give a maximum current density of $\approx 3$ $\times 10^{9} \mathrm{~A} / \mathrm{cm}^{2}$. Both values are roughly three orders of magnitude higher than for a typical metal.

On the first sight the wafer processed graphene/SiC material combination should be ideal for a systematic test of graphene's HC density behavior, potentially better suited than exfoliated graphene flakes on a wafer. We can routinely fabricate nearly identical samples in epitaxial quality. Hence, the influence of an irregular surface is reduced compared to deposited flakes. However, the material we use has a rather strong electron-phonon coupling to the substrate, which becomes apparent as a significant temperature dependence of the resistance. This is hypothetically due to rather soft phonons in the so called buffer layer, a nonconducting carbon $6 \sqrt{3} \times 6 \sqrt{3} \mathrm{R} 30^{\circ}$ reconstruction layer between the $\mathrm{SiC}$ bulk and the graphene layer.

${ }^{\text {a)} E l e c t r o n i c ~ m a i l: ~ h e i k o . w e b e r @ p h y s i k . u n i-e r l a n g e n . d e . ~}$
We prepared Hall bar structures of different sizes $(w=0.5-5 \mu \mathrm{m}, L=8-25 \mu \mathrm{m})$ by e-beam lithography [see for example Fig. 1(b)]. The process details are described in Ref. 2. The source and drain electrodes were built as large graphene/Ti/Au stacks to provide low contact resistances. For comparison, a reference sample with identical leads but without the graphene Hall bar has been fabricated [see Fig. $1(\mathrm{a})]$.

To study the effect of $\mathrm{HC}$ densities the samples are mounted inside a probe station. All measurements are performed with the chip at room temperature. The experimentation chamber is kept at helium temperature such that the vacuum is cryogenic. An excellent vacuum is mandatory: experiments under "only" high vacuum conditions revealed that large applied voltages result in the ignition of a local plasma which rapidly destroys the graphene sample. This requirement is similar to the reports in Ref. 4. In this clean environment we find two regimes with qualitatively different behavior. First for current densities $j=I / w \leqq 4 \mathrm{~mA} / \mu \mathrm{m}$ with the width of the Hall bar $w$ (corresponding to 1.3 $\left.\times 10^{9} \mathrm{~A} / \mathrm{cm}^{2}\right)$ a cleaning of the graphene is observed. We call this the current annealing (CA) regime. Second in the $\mathrm{HC}$ regime for $j=I / w>4 \mathrm{~mA} / \mu \mathrm{m}$ the sample glows locally and an irreversible process starts.

The experimental protocol is as follows: the bias voltage is applied until $I(t)$ is stable. Subsequently, the bias voltage is switched off and the sample is allowed to cool down to room

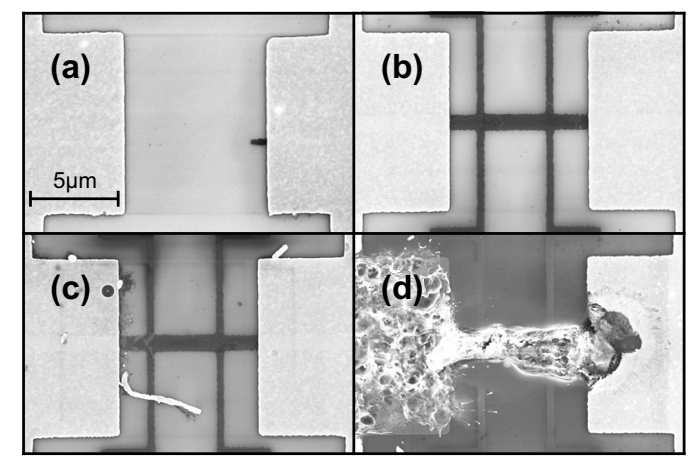

FIG. 1. SEM images of the presented samples: (a) reference structure with metallized graphene pads but the Hall bar is left out, (b) sample with width $1000 \mathrm{~nm}$ prior to measurement, (c) immediately before complete breakdown, and (d) after avalanche-like destruction. 


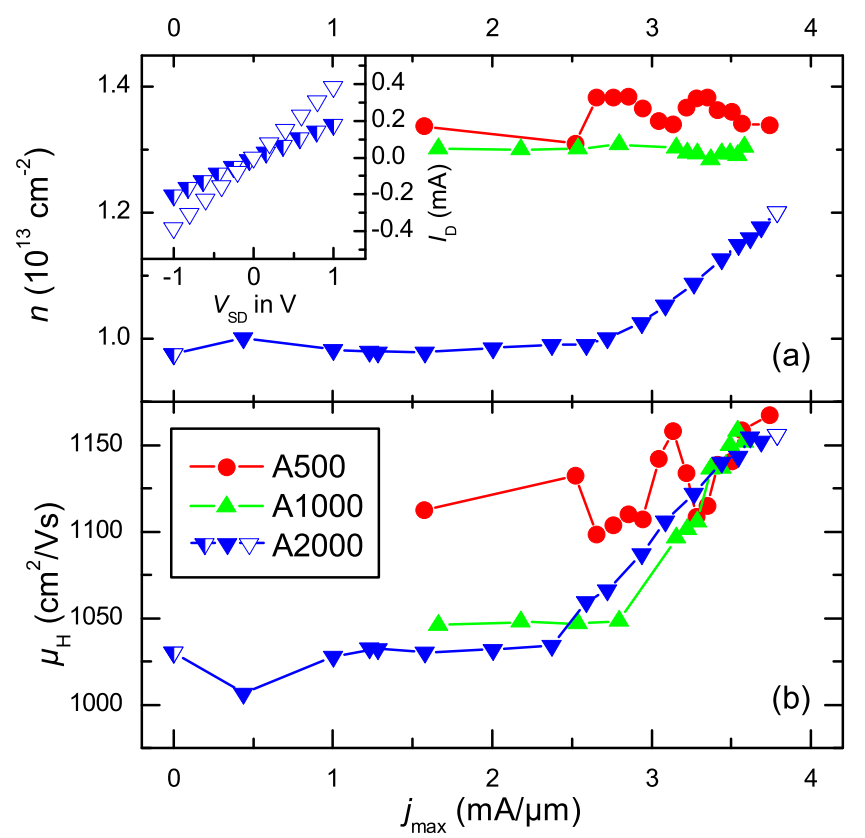

FIG. 2. (Color online) (a) Charge carrier density $n$ and (b) Hall mobility $\mu$ as a function of the previously seen current density $j_{\max }$ for three different samples with different channel widths between 500 and $2000 \mathrm{~nm}$. A clear enhancement in mobility can be seen in this CA regime. Inset: low bias $I-V$ characteristics of sample A2000 [symbols correspond to the respective symbols in (a) and (b)].

temperature. Then Hall resistance and conductivity are measured without breaking the cryogenic vacuum. This procedure is repeated for different voltages up to $U=200 \mathrm{~V}$.

We first discuss the CA regime. In Fig. 2 the CA of Hall bars with different widths between 0.5 and $2 \mu \mathrm{m}$ is shown. We have chosen to plot both $n$ and $\mu$ as derived from standard Hall effect evaluation (single charge carrier type) as a function of $j_{\max }$, the maximal current density the sample experienced before. It is clearly visible that upon the annealing process $n$ increases to $1.3 \times 10^{13} \mathrm{~cm}^{-2}$, which fits well to the value observed with $\mathrm{x}$-ray photoemission spectroscopy (XPS) and angle-resolved photoemission spectroscopy (ARPES) under ultra-high vacuum conditions (after the samples are annealed at $400{ }^{\circ} \mathrm{C}$ in situ). $\mu$ also increases which may indicate that the number of scattering centers is reduced when adsorbates are removed from the surface. Repeated measurements after exposing the sample several days to ambient atmosphere give the same results. Therefore, we think that the adsorbates stem rather from the ambient atmosphere than from lithography processing residues. The positive effect of CA on the transport properties was reported for exfoliated graphene before ${ }^{7,8}$ and has developed into a standard cleaning technique for suspended graphene sheets.

We further increased the applied voltage step by step and waited until the resulting current density reached a stable value $j_{\max }$. We now choose a different plot: in Fig. 3(a) the high bias conductivity is plotted over the actual $j_{\max }$. The data at high bias show a monotonously increasing current density, increasing power and, therefore, increasing temperature. At $j_{\max } \approx 4 \mathrm{~mA} / \mu \mathrm{m}$ and beyond the sample was glowing brightly. No significant discontinuity in $\sigma_{\text {hot }}\left(j_{\max }\right)$ is observed although certainly extreme conditions are reached.

There exists a very last data point which did not show convergence to a stable current plateau but rather a selfamplification of the current in time. This nonreversible

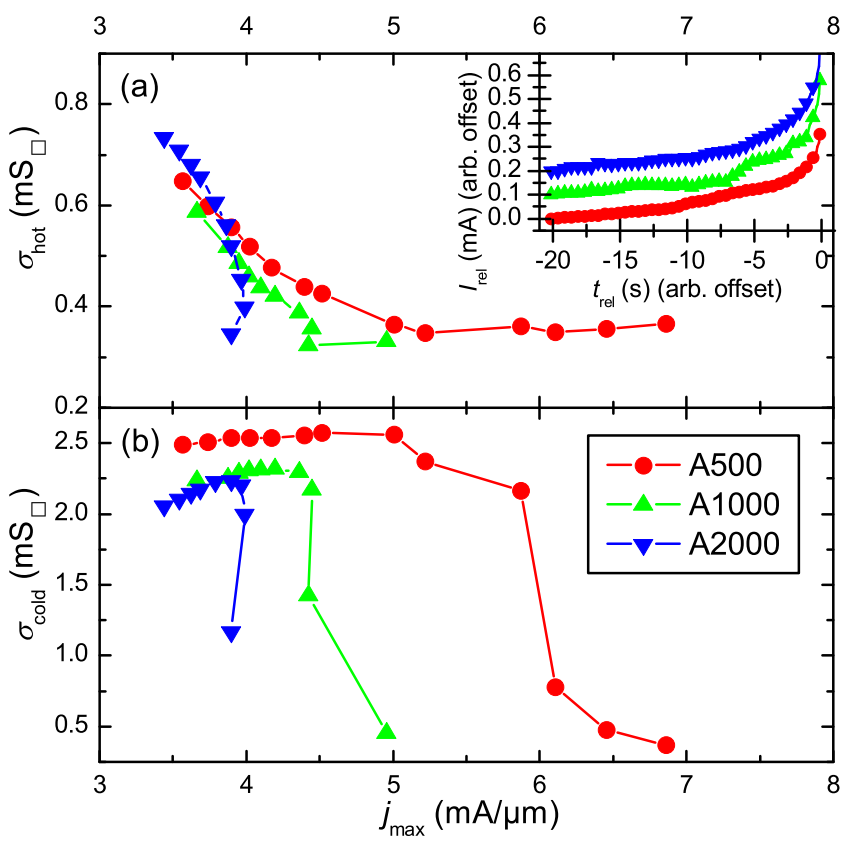

FIG. 3. (Color online) Conductivity $\sigma$ plotted as a function of $j_{\max }$ for different samples. (a) measurement while maximal current is flowing. The temperatures rise well beyond $1000{ }^{\circ} \mathrm{C}$. Inset: self-amplifying breakdown. (b) measurement after cooling to room temperature.

strong increase in the current was limited by the electronic circuit to $10 \mathrm{~mA}$ [see inset in Fig. 3(a)]. When we interrupted the current flow as soon as first indications of selfamplification occurred, the SEM picture [Fig. 1(c)] did not show significant change to the pristine sample. When, however, we allow for the self-amplification, the resulting SEM images look like Fig. 1(d), with large areas being vastly destroyed.

Although we did not measure the actual temperature we assume that the local temperature is substantially higher than $1200{ }^{\circ} \mathrm{C}$ as indicated by the bright white color of the glowing spot. We know, however, from the growth process in ultra high vacuum that from $1150{ }^{\circ} \mathrm{C}$ on growth of graphene is observed due to thermal decomposition of $\mathrm{SiC}$. Hence, one should expect the growth of graphitic layers under these conditions which add additional current paths to the bridge. This may explain both the self-amplification of the current as well as the damage pattern. An atomic force microscopy profile of the sample after destruction supports this notion: the damaged surface is lowered with respect to the unchanged areas.

Note that we observed no clear current breakdown in more than ten samples. From these data, one may conclude that with increasing current, the thermal decomposition of the substrate creates more and more current paths in addition to the graphene layer, instead of electromigration. This turns out to be questionable, when further data are considered: equivalent to the protocol in the CA regime, we recorded Hall data in between the voltage steps. For each point $j_{\max }$ was switched off, the sample was allowed for cooling to room temperature and the Hall measurement was carried out at low bias. Figure 3(b) displays the conductivity as a function of $j_{\max }$ : after the initial cleaning process explained before, the conductivity is rather constant up to $4-6 \mathrm{~mA} / \mu \mathrm{m}$, but then a sudden decrease in the longitudinal conductance indicates that the graphene has been damaged. A closer look at the Hall data yields that the charge carrier density remains 
essentially constant, while the charge carrier mobility drops down and is responsible for the drop in $\sigma$. We interpret this as the expected electromigration breakdown of the graphene layer.

There is, however, seemingly a contradiction between the continuous high-temperature data and the discontinuous low-temperature data. Note that the data in Figs. 3(a) and 3(b) represent the same measurement at different local temperature. The $\mathrm{SiC}$ substrate is fully insulating at room temperature; a value of $\approx 10 \mathrm{~T} \Omega$ up to $300 \mathrm{~V}$ has been determined in the reference sample [Fig. 1(a)]. Since the room temperature conductivity in Fig. 3(b) does not fully vanish, we have now apparently three materials: (i) the graphene layer with its electrical breakdown, (ii) the $\mathrm{SiC}$ substrate, which contributes only to the high- temperature conductivity, (iii) a material created out of the $\mathrm{SiC}$ substrate by the extreme temperature conditions, presumably a graphitic/ carbonic material of unknown composition. The disruption of the graphene conductance is invisible in the $\mathrm{HC}$ regime due to the dominating conductivity of the materials (ii) and (iii). The consequence is astonishing: epitaxial graphene has no visible current breakdown but rather reinforces the current flow. At the very last data point, the current (at constant voltage) induces thermal decomposition, leading to additional current paths. This mechanism self-amplifies to the avalanchelike behavior in Fig. 3(a) inset. The result is a complete destruction of the local device as shown in Fig. 1(d). We noted that in all samples the damage was concentrated on the more negative electrode. This may be explained by the different role of the two graphene/SiC Schottky contacts, one being forward biased and one being reverse biased. Due to the higher voltage drop, the latter dissipates more power. The current fraction running through the substrate thus triggers the decomposition avalanche on the more negative side.

In a further experiment, we repeated the measurement using short current pulses (duration $t_{\mathrm{p}}=1 \mathrm{~ms}$ ), instead of stabilization times which often reached minutes. Our initial notion was that thus heat becomes less dominant and momentum transfer by the current density should gain relative importance. Surprisingly, although the timescale was reduced by four orders of magnitude, no change in the overall behavior was observed. Also the maximum current density has very similar values.

In conclusion, we measured high quality epitaxial graphene on $\mathrm{SiC}$ under $\mathrm{HC}$ densities. For current densities below $j_{\max } \approx 4 \mathrm{~mA} / \mu \mathrm{m} \mathrm{CA}$ was observed. Both the charge carrier density as well as mobility are enhanced by this treatment. For larger current densities, very high temperatures are reached, observable as local bright glowing and irreversible decomposition of the substrate. Seemingly, the conductance increases more and more (due to additional carbonic material being formed) and the material reinforces itself under large current densities. A closer analysis shows that the SiC substrate and material formed by thermal decomposition of the substrate take over the current and the graphene film breaks down at a critical current density of $4-6 \mathrm{~mA} / \mu \mathrm{m}$. This value is approximately two times lower than the electromigration threshold reported for metallic nanotubes and three times larger than reported for exfoliated graphene. We assign the difference to the strong coupling to substrate phonons and the associated heating power in epitaxial graphene.

This work was carried out in the Cluster of Excellence "Engineering of Advanced Materials" at the FriedrichAlexander Universität Erlangen-Nürnberg.

${ }^{1}$ K. V. Emtsev, A. Bostwick, K. Horn, J. Jobst, G. L. Kellogg, L. Ley, J. McChesney, T. Ohta, S. A. Reshanov, J. Röhrl, E. Rotenberg, A. K. Schmid, D. Waldmann, H. B. Weber, and T. Seyller, Nature Mater. 8, 203 (2009).

${ }^{2}$ J. Jobst, D. Waldmann, F. Speck, R. Hirner, D. K. Maude, T. Seyller, and H. B. Weber, Phys. Rev. B 81, 195434 (2010).

${ }^{3}$ G. Pensl, F. Ciobanu, T. Frank, M. Krieger, S. Reshanov, F. Schmid, and M. Weidner, Int. J. High Speed Electron. Syst. 15, 705 (2005).

${ }^{4}$ B. Standley, W. Bao, H. Zhang, J. Bruck, C. N. Lau, and M. Bockrath, Nano Lett. 8, 3345 (2008).

${ }^{5}$ S. Hong and S. Myung, Nat. Nanotechnol. 2, 207 (2007).

${ }^{6}$ W. C. Marquardt, S. Grunder, A. Blaszczyk, S. Dehm, F. Hennrich, H. Löhneysen, M. Mayor, and R. Krupke, Nat. Nanotechnol. 5, 863 (2010).

${ }^{7}$ J. Moser, A. Barreiro, and A. Bachtold, Appl. Phys. Lett. 91, 163513 (2007).

${ }^{8}$ E. Cruz-Silva, A. R. Botello-Méndez, Z. M. Barnett, X. Jia, M. S. Dresselhaus, H. Terrones, M. Terrones, B. G. Sumpter, and V. Meunier, Phys. Rev. Lett. 105, 045501 (2010). 